\title{
Correlates of Referral Practices of General Surgeons to Plastic Surgeons for Mastectomy Reconstruction
}

\author{
Amy K. Alderman, $\mathrm{MD}, \mathrm{MPH}^{1,2}$ \\ Sarah T. Hawley, $\mathrm{PhD}^{2,3}$ \\ Jennifer Waljee, $\mathrm{MD}^{4}$ \\ Monica Morrow, MD ${ }^{5}$ \\ Steven J. Katz, MD, MPH ${ }^{2,3}$ \\ ${ }^{1}$ Section of Plastic Surgery, Department of Sur- \\ gery, the University of Michigan Medical Center, \\ Ann Arbor, Michigan. \\ ${ }^{2}$ Veterans Affairs Center for Practice Management \\ and Outcomes Research, Ann Arbor Veterans \\ Affairs Health Care System, Ann Arbor, Michigan. \\ ${ }^{3}$ Division of General Medicine, Department of In- \\ ternal Medicine, the University of Michigan Medi- \\ cal Center, Ann Arbor, Michigan. \\ ${ }^{4}$ Robert Wood Johnson Clinical Scholars Pro- \\ gram and Department of Surgery, the University \\ of Michigan Medical Center, Ann Arbor, Michigan. \\ ${ }^{5}$ Department of Surgical Oncology, Fox Chase \\ Cancer Center, Philadelphia, Pennsylvania.
}

Supported by a grant from the National Cancer Institute (R01 CA8837-A1) to the University of Michigan. This project has been funded in part with federal funds from the National Cancer Institute, National Institutes of Health, Department of Health and Human Services, under contract N01PC-35139 and N01-PC-65064. The collection of cancer incidence data used in this publication was supported by the California Department of Health Services as part of the statewide cancer reporting program mandated by California Health and Safety Code Section 103885.

We thank the American College of Surgeons Breast Cancer Department (Connie Bura and David Winchester, MD) for their support.

The ideas and opinions expressed herein are those of the author, and no endorsement by the State of California, Department of Health Services is intended or should be inferred.

Address for reprints: Amy K. Alderman, MD, MPH, Section of Plastic Surgery, Department of Surgery,

BACKGROUND. General surgeons' attitudes toward breast reconstruction may affect referrals to plastic surgeons. The propensity to refer to plastic surgeons prior to surgical treatment decisions for breast cancer varies markedly across general surgeons and is associated with receipt of reconstruction. In this study, the authors used data from a large physician survey to examine factors associated with general surgeons' propensity to refer breast cancer patients to plastic surgeons prior to mastectomy.

METHODS. The authors surveyed all attending general surgeons ( $\mathrm{N}=456$ surgeons) from a population-based sample of breast cancer patients who were diagnosed in Detroit and Los Angeles during 2002 ( $\mathrm{N}=1844$ patients), with a surgeon response rate of $80 \%$. The dependent variable was surgeon report of the percentage of their mastectomy patients in the past 2 years who they referred to plastic surgeons prior to initial surgery (referral propensity). Referral propensity was collapsed into 3 categories $(<25 \%, 25-75 \%$, and $>75 \%)$ and regressed on the following covariates using logistic regression: Surveillance, Epidemiology, and End Results registry; number of years in clinical practice; surgeons' sex; annual breast surgery volume; and hospital setting. RESULTS. Only $24 \%$ of surgeons referred $>75 \%$ of their patients to plastic surgeons prior to surgery (high referral propensity). High referral propensity was associated independently with surgeons who were women (odds ratio $[\mathrm{OR}], 2.3 ; P=.03$ ), high clinical breast surgery volume (OR, $4.1 ; P<.01)$, and working in cancer centers (OR, 2.4; $P=.01$ ). High-referral surgeons and low-referral surgeons also had different beliefs about women's preferences for reconstruction, with the low-referral surgeons perceiving more access barriers (cost, availability of plastic surgeons) and a lower patient priority for reconstruction.

CONCLUSIONS. A large proportion of surgeons do not refer breast cancer patients to plastic surgery at the time of surgical decision-making. Surgeons who have a high referral propensity are more likely to be women, to have a high clinical breast volume, and to work in cancer centers. These data support the importance of comanagement through multidisciplinary care models. Women need more opportunities to discuss reconstructive options to make informed surgical treatment decisions about their breast cancer. Cancer 2007;109:1715-20. ㄷ 2007 American Cancer Society.

KEYWORDS: breast reconstruction, practice patterns, surgical decision-making, Surveillance, Epidemiology, and End Results.

ess than $20 \%$ of eligible patients undergo breast reconstruction after treatment for breast cancer, despite documented qualityof-life benefits of the procedure. ${ }^{1-3}$ Considerably lower rates of reconstruction have been observed in many geographic regions and in nonwhite racial and ethnic subgroups, which has motivated con-

University of Michigan, 2130 Taubman Center, 1500 East Medical Center Drive, Ann Arbor, Ml 48109-0340; Fax: (734) 763-5354; E-mail: aalder@umich.edu
Received October 30, 2006; revision received January 12, 2007; accepted January 19, 2007. 
cerns about access to treatment. ${ }^{3}$ In response, the Women's Health and Cancer Rights Act (WHCRA) was passed in 1998: it is a federal law that mandates insurance coverage of breast reconstruction. ${ }^{4}$ However, the law has not increased the overall use of reconstruction or reduced variations across geographic regions and patient subgroups. ${ }^{5}$ This suggests that factors other than insurance coverage are driving practice patterns.

The literature has illuminated patient-level factors that are associated with use of reconstruction. Older, non-Caucasian women with more advanced cancer stage are less likely to receive breast reconstruction. ${ }^{3,6}$ In addition, compared with Caucasian women, African-American women are significantly more likely to report that reconstruction was not recommended to them or that they did not have enough knowledge to make an informed decision about it. ${ }^{6}$ Much less is known about provider and organizational factors associated with patients' receipt of breast reconstruction. Hawley et al. showed that a surgeon's propensity to refer their patients with breast cancer to a plastic surgeon prior to surgical cancer treatment explained a substantial amount of between-surgeon variation in the use of reconstruction. ${ }^{7}$ This suggested that surgeons' attitudes and practice factors may be important sources of variation in reconstruction. To examine these issues further, we used a surgeon survey that accompanied a large, population-based study 1) to describe surgeon and practice-level factors associated with a general surgeons' propensity to refer their breast cancer patients to a plastic surgeon for consultation about reconstruction prior to mastectomy and 2) to describe general surgeons' perceptions about why women do not seek breast reconstruction.

\section{MATERIALS AND METHODS \\ Study Population}

We performed a survey of a population-based sample of 2645 women with breast cancer who were diagnosed in the metropolitan areas of Detroit and Los Angeles during the period from December 2001 to January 2003. The details of this study are reported elsewhere. ${ }^{8}$ In brief, all patients with ductal carcinoma in situ and an approximate $20 \%$ random sample of patients with invasive (but not metastatic) disease were accrued into the sample during the study period. Pathology reports were used to identify 1 or more surgeons ( $\mathrm{N}=456$ surgeons) for $98.5 \%$ of the patient sample.

Surgeons were contacted by mail and were asked to participate in a brief, self-administered survey to evaluate their perspectives about the surgical treatment for breast cancer. Surgeons subsequently were mailed a questionnaire and a gift. We followed the Dillman method to maximize response rates in the mailed survey, ${ }^{9,10}$ which involved a postcard reminder and subsequent mailings to nonresponders. The response rate was $80 \%$ ( $\mathrm{N}=365$ surgeons): Three hundred fifty-five surgeons completed the written questionnaire, and 10 surgeons completed an abbreviated telephone survey. Surgeons were informed about the study goals and methods, and the protocol was approved by the Institutional Review Boards of the University of Michigan, Wayne State University, and the University of Southern California.

\section{Measures}

The dependent variable was referral propensity to plastic surgery prior to mastectomy. Surgeons were asked to indicate the percentage of their patients in the past 2 years who were considering mastectomy who they referred to a plastic surgeon prior to initial surgery $(<25 \%, 25 \%$ to $49 \%, 50 \%$ to $75 \%$, or $>75 \%)$. The middle 2 groups were combined in this analysis to create 3 categories of referral propensity: low, moderate, and high. For the logistic regression, we categorized referral propensity further into low (low and moderate) versus high.

The independent variables included 1) Surveillance, Epidemiology, and End Results (SEER) registry (Detroit vs Los Angeles); 2) surgeon characteristics (sex, years in practice since residency); 3) surgeon volume (the number of definitive breast cancer surgery procedures per year); and 4) hospital practice setting (cancer center, teaching hospital, community hospital). Years in practice was self-reported and was analyzed as a continuous variable. To measure clinical breast volume, we recoded the reported number of total breast procedures in the past year into 3 categories of near equal distribution: low $(<20$ procedures per year), medium (21-50 procedures per year), and high ( $>50$ procedures per year). Varying the cutoff ranges did not substantively change the results. Surgeon practice affiliation was categorized as a cancer center, teaching hospital, or community hospital based on surgeon self-report. Because surgeons could choose more than 1 practice setting, we applied the following algorithm to assign surgeons to a primary setting: cancer center (alone or with either teaching or community setting), teaching setting (teaching hospital without cancer center but with or without community hospital), or community hospital (with community hospital the only setting category indicated). 


\section{Analysis}

First, we described the distribution of referral propensity (3 categories) across all independent variables. Then, we regressed surgeon referral propensity (2 categories) on selected covariates using logistic regression. Wald tests and the likelihood-ratio test were used to test the significance of individual predictive variables, and the model chi-square statistic was applied to test the overall significance of the model. All analyses were performed with STATA software (version 8.0).

\section{RESULTS}

Table 1 shows the study sample characteristics. The mean surgeon age was 49.4 years, $14.4 \%$ of surgeons were women, and they had a mean of 17.2 years in practice. On average, surgeons reported that they devoted $31.3 \%$ of their total practice to breast cancer. There was a wide distribution of annual procedure volume by surgeon: $28.5 \%$ reported that they performed $\leq 20$ definitive breast surgeries per year; and $31.0 \%$ performed $>50$ procedures per year. Approximately $50 \%$ of surgeons practiced in a community hospital setting alone, 33\% practiced in a teaching hospital, and $18.8 \%$ practiced in a cancer center.

Table 2 describes the distribution of general surgeons' referral propensity to plastic surgery for breast reconstruction across selected demographic and clinical variables. SEER registry, surgeon sex, procedure volume, and hospital setting were associated significantly with referral practice. Table 3 shows the independent association of covariates with referral propensity categories. High-referral surgeons were more likely to be women (odds ratio [OR], 2.30; $P=.03$ ), to have high clinical breast surgery volume (OR, 4.08; $P<.01$ ), and to work in cancer centers (OR, 2.41; $P=.01$ ).

Table 4 describes general surgeons' beliefs about why women do not receive breast reconstruction. Many surgeons believed that patients did not undergo reconstruction because of a lack of patient desire. Specifically, $57 \%$ of surgeons believed that reconstruction was not important to patients; $64 \%$ believed that patients were not interested; and $39 \%$ believed that patients were concerned that reconstruction would take too long. However, nearly half of surgeons $(46 \%)$ reported that patients were concerned about the cost of the procedure. Table 4 also describes the distribution of surgeon beliefs about why women do not receive breast reconstruction across referral propensity categories. There were few differences across referral propensity categories in surgeon beliefs related to patient desire for reconstruction.
TABLE 1

Study Sample Characteristics $(\mathrm{N}=365)$

\begin{tabular}{|c|c|c|}
\hline Variable & No. & Percentage \\
\hline \multicolumn{3}{|l|}{ Site } \\
\hline Detroit & 138 & 37.8 \\
\hline Los Angeles & 227 & 62.2 \\
\hline Mean age (range), y & $49.4(30-79)$ & \\
\hline Years in practice, mean (range) & $17.2(1-49)$ & \\
\hline$\leq 10$ & 108 & 30.2 \\
\hline $11-20$ & 115 & 32.1 \\
\hline $21-49$ & 135 & 37.7 \\
\hline \multicolumn{3}{|l|}{ Sex } \\
\hline Women & 53 & 14.4 \\
\hline Men & 312 & 85.6 \\
\hline \multicolumn{3}{|l|}{ Race } \\
\hline White & 237 & 64.9 \\
\hline Black & 15 & 4.1 \\
\hline Hispanic & 16 & 4.4 \\
\hline Asian & 70 & 19.2 \\
\hline Other & 27 & 7.4 \\
\hline \multicolumn{3}{|l|}{ Hospital practice setting } \\
\hline Cancer center & 69 & 18.8 \\
\hline Teaching hospital & 120 & 33 \\
\hline Community hospital & 176 & 48.2 \\
\hline \multicolumn{3}{|l|}{ No. of breast surgeries per y } \\
\hline$\leq 20$ & 104 & 28.5 \\
\hline $21-50$ & 135 & 37 \\
\hline$>50$ & 113 & 31 \\
\hline Missing & 13 & 3.5 \\
\hline Mean percentage of breast cancer surgeries (range) & & $31.3(2-100)$ \\
\hline Percentage other oncology & & $16.2(0-70)$ \\
\hline Percentage nononcology & & $52.5(0-95)$ \\
\hline
\end{tabular}

Similarly, there was little difference across referral propensity categories in surgeon beliefs that patients commonly voiced concerns that reconstruction would take too long. By contrast, there were marked differences across surgeon referral propensity categories in surgeon beliefs related to potential patient access barriers, such as inadequate knowledge $(32 \%, 16 \%$, and $12 \%$ for low, moderate, and high referral propensity categories, respectively; $P<.001$ ), concerns about cost (58\%, $47 \%$, and $22 \%$ for low, moderate, and high referral propensity categories, respectively; $P<.001$ ), and unavailability of plastic surgeons $(30 \%, 13 \%$, and $8 \%$ for low, moderate, and high referral propensity categories, respectively; $P<.001)$. There also were marked differences across propensity referral categories in surgeon beliefs about patient priorities for treatment. In particular, $31 \%, 13 \%$, and $12 \%$ of surgeons in the low, moderate, and high referral propensity categories, respectively $(P<.001)$, believed that patients were too preoccupied with other elements of their cancer therapy to consider reconstruction.

Several other themes emerged from an open ended question about why women do not choose 
TABLE 2

Distribution of Plastic Surgery Referral Propensity Across Selected Surgeon Characteristics $(\mathrm{N}=342)^{*}$

\begin{tabular}{|c|c|c|c|c|}
\hline \multirow[b]{2}{*}{ Variable } & \multicolumn{3}{|c|}{ Percentage of surgeons } & \multirow[b]{2}{*}{$\boldsymbol{P}^{\dagger}$} \\
\hline & $\begin{array}{l}\text { Low-referral } \\
\text { surgeons, } n=152\end{array}$ & $\begin{array}{l}\text { Moderate-referral } \\
\text { surgeons, } \mathrm{n}=109\end{array}$ & $\begin{array}{l}\text { High-referral } \\
\text { surgeons, } n=81\end{array}$ & \\
\hline \multicolumn{4}{|l|}{ SEER registry } & .001 \\
\hline Detroit & 53 & 31 & 31 & \\
\hline Los Angeles & 47 & 69 & 69 & \\
\hline \multicolumn{4}{|l|}{ Years in practice } & .23 \\
\hline$\leq 10$ & 24 & 32 & 38 & \\
\hline $11-20$ & 32 & 32 & 29 & \\
\hline $21-49$ & 44 & 36 & 33 & \\
\hline \multicolumn{4}{|l|}{ Sex } & $<.001$ \\
\hline Men & 95 & 82 & 70 & \\
\hline Women & 5 & 18 & 30 & \\
\hline \multicolumn{4}{|l|}{ Clinical breast volume } & $<.001$ \\
\hline Low & 33 & 35 & 11 & \\
\hline Medium & 42 & 35 & 38 & \\
\hline High & 25 & 30 & 51 & \\
\hline \multicolumn{4}{|l|}{ Hospital setting } & $<.001$ \\
\hline Community & 51 & 33 & 34 & \\
\hline Teaching & 25 & 42 & 35 & \\
\hline Cancer center & 9 & 22 & 30 & \\
\hline \multicolumn{5}{|c|}{ SEER indicates Surveillance, Epidemiology, and End Results. } \\
\hline \multicolumn{5}{|c|}{ * Twenty-three surgeons had missing data on referral practice. } \\
\hline \multicolumn{5}{|c|}{ † Pearson chi-square test for differences in the distribution of referral propensity across the selected variables. } \\
\hline \multicolumn{5}{|c|}{$\begin{array}{l}\text { * Low-referral surgeons referred }<25 \% \text { of breast cancer patients to a plastic surgeon prior to mastectomy; moderate-referral surgeons referred } 25-75 \% \text { of } \\
\text { patients; and high-referral surgeons referred }>75 \% \text { of patients. }\end{array}$} \\
\hline
\end{tabular}

TABLE 3

Multivariate Analysis of Correlates of a High-Referral General Surgeon*

\begin{tabular}{|c|c|c|}
\hline Independent variable & Adjusted OR (95\% CI) & $P$ \\
\hline SEER registry ${ }^{\dagger}$ & $1.50(0.81-2.76)$ & .20 \\
\hline Years in practice & $0.99(0.96-1.02)$ & .40 \\
\hline Women ${ }^{\S}$ & $2.30(1.09-4.84)$ & .03 \\
\hline \multicolumn{3}{|l|}{ Clinical breast volume } \\
\hline Low ${ }^{\|}$ & 1.0 & - \\
\hline Medium & $2.98(1.30-6.82)$ & .01 \\
\hline High & $4.08(1.76-9.42)$ & $<.01$ \\
\hline$F$ test $($ chi-square $=10.9)$ & & $<.01$ \\
\hline \multicolumn{3}{|l|}{ Hospital setting } \\
\hline Community hospitall & 1.0 & - \\
\hline Teaching hospital & $1.73(0.89-3.36)$ & .10 \\
\hline Cancer center & $2.41(1.16-5.04)$ & .01 \\
\hline$F$ test $($ chi-square $=5.6)$ & & .05 \\
\hline
\end{tabular}

OR indicates odds ratio; 95\% CI, 95\% confidence interval; SEER, Surveillance, Epidemiology, and End Results.

${ }^{*}$ High-referral surgeons were defined as those who referred $>75 \%$ of breast cancer patients to plastic surgery for reconstruction.

$\dagger$ Detroit was the reference group.

\$ Continuous variable.

${ }^{\S}$ Men were the reference group.

" Reference group. reconstruction: poor reconstructive options (specifically, the abdominal wall complications with transverse rectus abdominis musculocutaneous flaps, the fear of silicone, and the poor cosmetic results with breast implants), the risk that reconstruction may delay adjuvant therapy, and the social stigma of having a cosmetic procedure. In addition, several surgeons mentioned difficulty with health maintenance organization coverage of procedures despite local and federal legislation and patient advocacy letters.

\section{DISCUSSION}

In this large, population-based study, we observed that almost half of surgeons (44\%) referred $<25 \%$ of women to plastic surgery prior to the mastectomy. High-referral surgeons were more likely to be women, to have a high clinical breast surgery volume, and to work in cancer centers. Surgeon attitudes about why women do not receive breast reconstruction varied systematically. Low-referral surgeons were more likely to perceive access barriers to receiving reconstruction and lower patient priorities for reconstruction; but there was little difference across surgeons with 
TABLE 4

Surgeons' Perceptions of Why Women Do Not Chose Breast Reconstruction by Surgeon Referral Practice to Plastic Surgery for Breast Reconstruction

\begin{tabular}{|c|c|c|c|c|c|}
\hline \multirow[b]{2}{*}{ Surgeons' perception } & \multicolumn{4}{|c|}{ Responded "very common," \%* } & \multirow[b]{2}{*}{$p^{\|}$} \\
\hline & $\begin{array}{l}\text { All surgeons, } \\
\mathrm{N}=342^{\dagger}\end{array}$ & $\begin{array}{l}\text { Low-referral } \\
\text { surgeons, } \mathrm{n}=152\end{array}$ & $\begin{array}{l}\text { Moderate-referral } \\
\text { surgeons, } \mathrm{n}=109\end{array}$ & $\begin{array}{l}\text { High-referral } \\
\text { surgeons, } \mathrm{n}=81\end{array}$ & \\
\hline \multicolumn{6}{|l|}{ Patient desire } \\
\hline No desire for more surgery & 64 & 62 & 70 & 60 & .32 \\
\hline Not important & 57 & 58 & 58 & 53 & .67 \\
\hline Requires too much time & 39 & 30 & 51 & 40 & $<.01$ \\
\hline \multicolumn{6}{|l|}{ Patient concern } \\
\hline Concerned about cancer surveillance & 25 & 24 & 30 & 19 & .19 \\
\hline $\begin{array}{l}\text { Concerned about the look or feel } \\
\text { of the reconstruction }\end{array}$ & 15 & 16 & 17 & 9 & .21 \\
\hline \multicolumn{6}{|l|}{ Access barriers } \\
\hline Concerned about cost & 46 & 58 & 47 & 22 & $<.001$ \\
\hline $\begin{array}{l}\text { Not enough knowledge about } \\
\text { reconstruction }\end{array}$ & 22 & 32 & 16 & 12 & $<.001$ \\
\hline Unavailability of plastic surgery & 19 & 30 & 13 & 8 & $<.001$ \\
\hline \multicolumn{6}{|l|}{ Patient priorities } \\
\hline $\begin{array}{l}\text { Focused on breast cancer } \\
\text { treatment, not reconstruction }\end{array}$ & 21 & 31 & 13 & 12 & $<.001$ \\
\hline \multicolumn{6}{|c|}{ * Responded 4 or 5 on a 5-point Likert scale, from very uncommon (1) to very common (5). } \\
\hline \multicolumn{6}{|c|}{$\begin{array}{l}\ddagger \text { Low-referral surgeons referred }<25 \% \text { of breast cancer patients plastic surgeon prior to mastectomy; moderate-referral surgeons referred } 25-75 \% \text { of patients; and high-referral surgeons referred }>75 \% 0 \\
\text { patients. }\end{array}$} \\
\hline
\end{tabular}

regard to their perceptions of patient desire for the treatment.

We can only speculate about whether these differences in referral practices across surgeons are driven by patient preferences, surgeons' attitudes toward treatment options, or practice barriers to comanagement. Results pertaining to surgeons' attitudes suggest that practice barriers may play an important role. Compared with high-referral surgeons, lowreferral surgeons were significantly more likely to perceive that patient finances and availability of reconstructive services diminished opportunities for reconstruction. These attitudes may indicate that low-referral surgeons are more likely to treat patients with limited resources or to practice in settings with limited access to plastic surgeons. Our observation that, compared with higher referral surgeons, lowreferral surgeons were more likely to perceive that low patient knowledge was a reason they did not receive reconstruction suggests that patient education level and/or resources devoted to patient education may be lower in their practices. The finding that low-referral surgeons were more likely to believe that women were more focused on other cancer treatments than reconstruction suggests that resources devoted to coordination of treatment may be more limited in these practices. By contrast, there were no differences across surgeons in beliefs about the role of patient desire in the receipt of reconstruction. This suggests that patient interest in reconstruction may be similar across surgeon practices.

\section{Limitations}

Our findings should be interpreted in the context of some limitations. Our estimates may have been biased by nonresponse, and we had little information about nonresponding surgeons. However, the response rate of $80 \%$ was excellent for a physician survey. We believe that the surgeon sample is representative of all surgeons practicing breast cancer care in the 2 metropolitan areas during the study period, because they were identified through a population-based sample of patients. However, we likely missed some surgeons, particularly those with a low breast surgery volume. These results also are limited to the 2 metropolitan areas and may not reflect national trends in breast cancer care. In addition, practice information was derived from self-report and is subject to respondent recall. However, surgeons' recall of procedure volume was correlated highly with the number of patients treated in our patient study. In addition, this study addresses referral to a plastic surgeon at the time of breast cancer diagnosis and does not include 
surgeon referrals after the initial course of breast cancer treatment. Finally, we do not have information on hospital practice attributes, such as patient volume, or patient attributes, such as stage of disease, which may be independent predictors of reconstructive referral practices.

\section{Implications}

Our results have important implications for patient care and policy. Prior research suggests that low rates of breast reconstruction reflect unmet need, especially in vulnerable populations. Our findings indicate that there are systematic differences among surgeons with regard to referral to plastic surgeons prior to surgical decisions for patients with breast cancer. Patient decision aids that include information about reconstruction ${ }^{11}$ or comanagement of patients through a multidisciplinary approach may improve patient knowledge about all surgical options and aid in this complex decision-making process. Referral to a plastic surgeon prior to initial surgical decision also may influence this decision; for example, women may be more inclined to choose mastectomy with a good understanding of the reconstructive options. However, our results suggest that barriers to comanagement may exist, especially in smaller surgical practices, which may have a more challenging patient mix and limited resources. The acceptance of multidisciplinary breast cancer treatment as a practice model, coupled with advances in breast reconstruction in the past 2 decades, should motivate strategies to enhance the involvement of plastic surgeons in the education and treatment counseling of patients with newly diagnosed breast cancer.

\section{REFERENCES}

1. Wilkins EG, Cederna PS, Lowery JC, et al. Prospective analysis of psychosocial outcomes in breast reconstruction: one-year postoperative results from the Michigan Breast Reconstruction Outcome Study. Plast Reconstr Surg. 2000; 106:1014-1025.

2. Cederna PS, Yates WR, Chang P, Cram AE, Ricciardelli EJ. Postmastectomy reconstruction: comparative analysis of the psychosocial, functional, and cosmetic effects of transverse rectus abdominis musculocutaneous versus breast implant reconstruction. Ann Plast Surg. 1995;35:458468.

3. Alderman AK, McMahon L, Wilkins EG. The national utilization of immediate and early delayed breast reconstruction and the impact of sociodemographic factors. Plast Reconstr Surg. 2003;11:695-703.

4. United States Department of Health and Human Services. Women's Health and Cancer Rights Act. Available at URL: http://www.cms.hhs.gov/hipaa/hipaal/content/whcra.asp Accessed June 8, 2005.

5. Alderman AK, Wei Y, Birkmeyer JD. Use of breast reconstruction after mastectomy following the Women's Health and Cancer Rights Act. JAMA. 2006;295:387-388.

6. Morrow M, Mujahid M, Lantz PM, et al. Correlates of breast reconstruction. Results from a population-based study. Cancer. 2005;104:2340-2346.

7. Hawley ST, Hofer TP, Janz NK, et al. Correlates of betweensurgeon variation in breast cancer treatments. Med Care. 2006;44:609-616.

8. Katz SJ, Lantz PM, Janz NK, et al. Patient involvement in surgery treatment decisions for breast cancer. J Clin Oncol. 2005;23:5526-5533.

9. Anema MG, Brown BE. Increasing survey responses using the total design method. J Contin Educ Nurs. 1995;26:109114.

10. Dillman D. Mail and Telephone Surveys. New York, NY: John Wiley \& Sons, Inc.; 1978.

11. Waljee JF, Rogers MAM, Alderman AK. Decision aids among women with early breast cancer: do they influence surgical choice? J Clin Oncol. 2007;25:1067-1073. 